\title{
Differentiation of Low-Grade Astrocytoma From Anaplastic Astrocytoma Using Radiomics-Based Machine Learning Techniques
}

\author{
Boran Chen ${ }^{1+}$, Chaoyue Chen ${ }^{1,2+}$, Jian Wang ${ }^{3}$, Yuen Teng ${ }^{1}$, Xuelei Ma ${ }^{2,4 *}$ \\ and Jianguo $\mathrm{Xu}^{1 *}$
}

${ }^{1}$ Department of Neurosurgery, West China Hospital, Sichuan University, Chengdu, China, ${ }^{2}$ State Key Laboratory of Biotherapy and Cancer Center, West China Hospital, Sichuan University, and Collaborative Innovation Center for Biotherapy, Chengdu, China, ${ }^{3}$ School of Computer Science, Nanjing University of Science and Technology, Nanjing, China,

${ }^{4}$ Department of Biotherapy, Cancer Center, West China Hospital, Sichuan University, Chengdu, China

\section{OPEN ACCESS}

Edited by:

Meiyappan Solaiyappan,

Johns Hopkins University,

United States

Reviewed by:

Guolin Ma,

China-Japan Friendship Hospital,

China

Andrea Hawkins-Daarud, Mayo Clinic Arizona, United States

*Correspondence:

Xuelei Ma

drmaxuelei@gmail.com

Jianguo $X u$

drijanguoxu@gmail.com

${ }^{\dagger}$ These authors have contributed equally to this work

Specialty section:

This article was submitted to

Cancer Imaging and Image-directed Interventions,

a section of the journal

Frontiers in Oncology

Received: 18 December 2019

Accepted: 04 May 2021

Published: 01 June 2021

Citation:

Chen B, Chen C, Wang J, Teng Y, $M a X$ and $X u J$ (2021) Differentiation of Low-Grade Astrocytoma From Anaplastic Astrocytoma Using Radiomics-Based Machine Learning Techniques.

Front. Oncol. 11:521313. doi: 10.3389/fonc.2021.521313
Purpose: To investigate the diagnostic ability of radiomics-based machine learning in differentiating atypical low-grade astrocytoma (LGA) from anaplastic astrocytoma (AA).

Methods: The current study involved 175 patients diagnosed with LGA $(n=95)$ or AA ( $n=$ 80) and treated in the Neurosurgery Department of West China Hospital from April 2010 to December 2019. Radiomics features were extracted from pre-treatment contrastenhanced T1 weighted imaging (T1C). Nine diagnostic models were established with three selection methods [Distance Correlation, least absolute shrinkage, and selection operator (LASSO), and Gradient Boosting Decision Tree (GBDT)] and three classification algorithms [Linear Discriminant Analysis (LDA), Support Vector Machine (SVM), and random forest (RF)]. The sensitivity, specificity, accuracy, and areas under receiver operating characteristic curve (AUC) of each model were calculated. Diagnostic ability of each model was evaluated based on these indexes.

Results: Nine radiomics-based machine learning models with promising diagnostic performances were established. For LDA-based models, the optimal one was the combination of LASSO + LDA with AUC of 0.825. For SVM-based modes, Distance Correlation + SVM represented the most promising diagnostic performance with AUC of 0.808. And for RF-based models, Distance Correlation + RF were observed to be the optimal model with AUC of 0.821.

Conclusion: Radiomic-based machine-learning has the potential to be utilized in differentiating atypical LGA from AA with reliable diagnostic performance.

Keywords: machine learning, glioma, astrocytoma, texture analysis, radiomics

\section{INTRODUCTION}

Astrocytoma is one of the most common intracranial tumors characterized by rapid evolvement emphasizing the challenge for early diagnosis and intervention (1). Based on the 2016 World Health Organization (WHO) Classification of Tumors of the Central Nervous System, astrocytoma could be classified into low-grade astrocytoma (LGA, WHO II) and anaplastic astrocytoma (AA, also 
known as high-grade astrocytoma, WHO III) (2). The treatment and prognosis of these two subtypes are dramatically different. LGA is recognized as the subtype with good prognosis for most patients, while AA is the one with poor prognosis that the 5-year overall survival only reaches $28 \%$ when receiving conventional treatments (3-6).

The serum marker for the grading of astrocytoma has not been identified yet. As for radiological examination, brain magnetic resonance imaging (MRI) has been highly recommended with the ability to sensitively detect small lesions and accurately localize lesions. A common differentiator on the contrast-enhanced T1 weight imaging (T1C) is the presence of enhancement of AA and the absence of enhancement of LGA. However, high-grade gliomas can sometimes mimic the appearance of low-grade gliomas, and enhancement can also be seen in some cases of low-grade gliomas which indicates aggressive behavior (7-10). The meta-analysis by Abrigo and colleagues included seven studies with 115 solid and non-enhancing glioma patients, and evaluated the diagnostic capability of cerebral blood volume (CBV), which was expressed as ratio of tumoral CBV to normal white matter CBV (rCBV), in differentiating low-grade gliomas and high-grade gliomas (11). Results of this meta-analysis indicated that $7 \%$ to $34 \%$ low-grade glioma cases may be misdiagnosed as high-grade gliomas, and around half of high-grade glioma cases may be misdiagnosed as low-grade gliomas by a rCBV threshold of 1.75 (11). The interpretation of MRI may be challenging and must take into account the timing of surgery, previous radiation, corticosteroid use, and chemotherapy (12). Additionally, three quarters of AA are the consequences of LGA transformation, which leads to the similarity on images and makes the presurgical distinctiveness of LGA from AA difficult in some cases (13). Thus, this highlights the urgent requirement of novel technology to make the interpretation of MRI more accurate and reliable.

Radiomics is the method which can provide non-visual information by extracting quantitative texture features with mathematical formulas. Moreover, with quantitative statistics extracted from images, machine learning algorithms could be introduced in assisting clinical practitioners in their work, such as diagnostic differentiation, clinical grading, and prognosis prediction (14-16). Thus, in the current study, we introduced the radiomicsbased machine learning algorithms in distinguishing atypical LGA from AA. The discriminative models were established with a set of texture parameters extracted from conventional MR images, and their diagnostic performances were evaluated for direct comparison.

\section{METHODS}

\section{Patient Selection}

This study was performed in the Department of Neurosurgery and the Cancer Centre of West China Hospital. We initially reviewed the electronic medical records from April 2010 to December 2019 in our institution. The inclusion criteria of patients were as follows: 1) pathologically confirmation of LGA or AA by intraoperative frozen-section reports; 2) available high-quality pre-treatment MR images performed in the Department of Radiology. Exclusion criteria were: 1) recorded history of other types of intracranial diseases, such as head trauma, brain tumor, subarachnoid hemorrhage, cerebral apoplexy, and ischemic infarction; 2) incomplete electronic medical records; 3) patients had underwent treatment, such as surgery, radiotherapy and chemotherapy, prior to available MR images. The clinical parameters were also recorded, including age, gender, and pathology reports; and the presurgical MR images were exported from Picture Archiving and Communication Systems (PACS). The workflow of the current research is shown in Figure 1.

\section{MR Image Acquisition}

Radiomics parameters should be extracted from the optimal MR sequence, defined as routine radiology examination which can provide precise segmentation of tumor tissue boundary. Therefore, T1C on the conventional MR sequence was selected. Other sequences like T1 weighted image (T1WI), T2 weighted image (T2WI), and fluid-attenuated inversion recovery (FLAIR) image were abandoned due to vague segmentation.

All patients underwent pre-treatment MR scan in the Department of Radiology in our institution with the 3.0T Siemens Trio Scanners before surgery. MPRAGE sequence was chosen to obtain high-resolution three-dimensional T1- weighted images. The parameters of scanners were: TR/TE/TI = 1900/2.26/ $900 \mathrm{~ms}$, slice thickness $=1 \mathrm{~mm}$, axial FOV $=25.6 \times 25.6 \mathrm{~cm}^{2}$, Flip angle $=9^{\circ}$, and data matrix $=256 \times 256$. Gadopentetate dimeglumine $(0.1 \mathrm{mmol} / \mathrm{kg})$ was used as the contrast agent for contrast-enhanced images. The multi-directional data of $\mathrm{T} 1 \mathrm{C}$ were collected during the continuous interval time of 90 to $250 \mathrm{~s}$. Figure 2 shows two examples of T1C sequence.

\section{Radiomics Analysis of MR Images}

Two neurosurgeons participated in the texture features extraction using LifeX package (http://www.lifexsoft.org) and following the instructions on the website (17). The regions of interest (ROI) along the boundary of lesions in each layer were drown by these two neurosurgeons to obtain the 3D-based texture features. Necrosis and vessels within tissue were also included in ROI. The separation between adjacent structure invasion and peritumoral edema band was carefully identified from the primary tumor with the different pattern in contrast enhancement. The texture features were calculated automatically from two orders with default software protocol settings. In the first order, the calculation was based on shape- and histogram-based matrices; and in the second order, the calculation was based on grey-level co-occurrence matrix (GLCM), grey-level zone length matrix (GLZLM), neighborhood grey-level dependence matrix (NGLDM), and grey-level run length matrix (GLRLM), which plays a major role in the quantification of radiomic features. Finally, a total number of 40 features were extracted for further deployment in machine learning algorithms.

\section{Human Reader Assessment}

To evaluated the diagnostic performance of human readers with naked eyes, two researchers independently made diagnosis of AA or LGA based on the contrast-enhancement pattern on T1C images and their experience. The human readers were blinded to the patient characteristics and the pathological reports. The diagnostic accuracy of LGA and AA and the overall diagnostic accuracy were calculated for further analysis. 


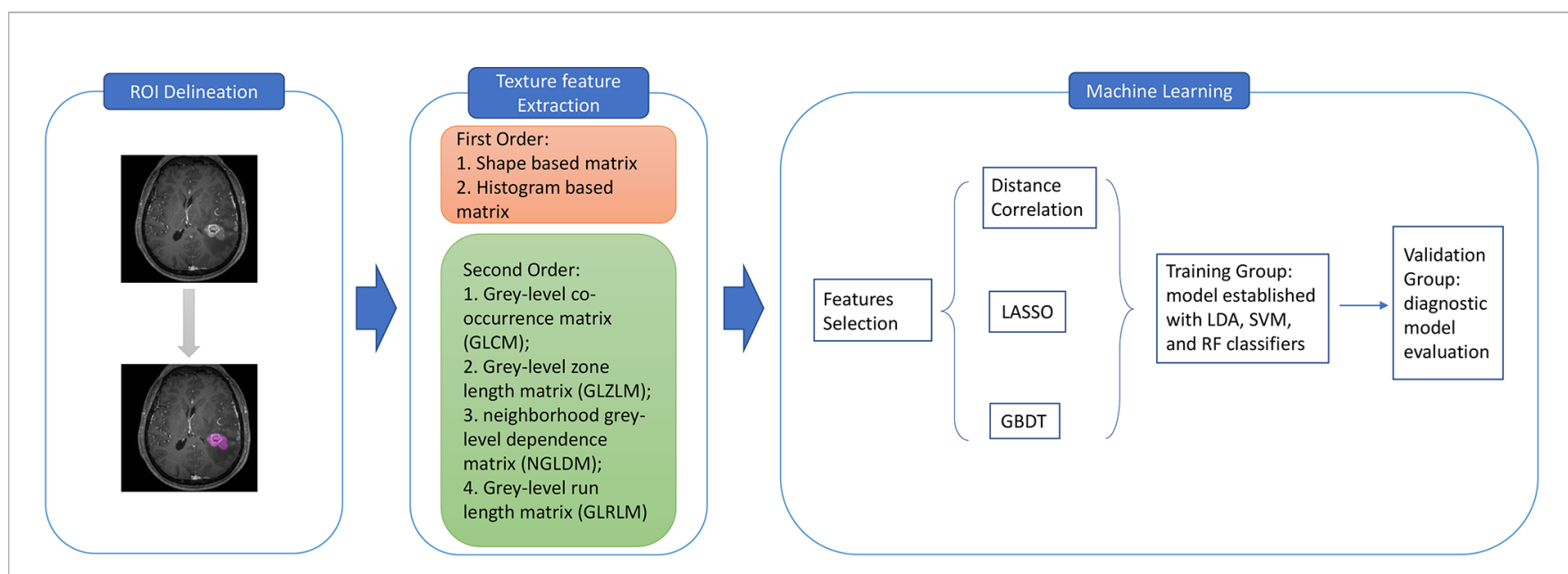

FIGURE 1 | The workflow for texture features extraction and machine learning classification. ROI, region of interest; GLCM, grey-level co-occurrence matrix; GLZLM, grey-level zone length matrix; NGLDM, neighborhood grey-level dependence matrix; GLRLM, grey-level run length matrix; LASSO, least absolute shrinkage and selection operator; GBDT, gradient correlation decision tree; LDA, linear discriminant analysis; SVM, support vector machine; RF, random forest.

A

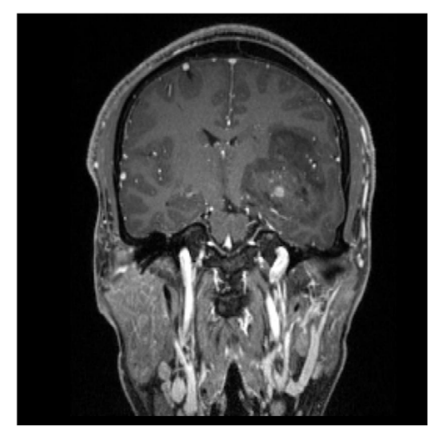

B

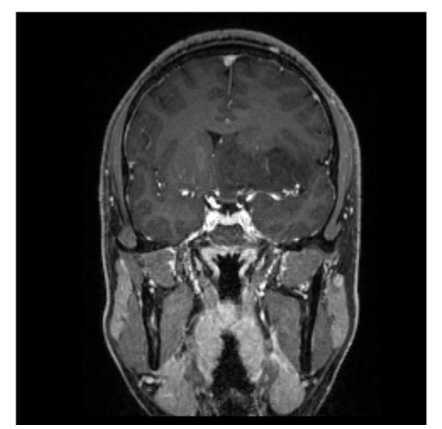

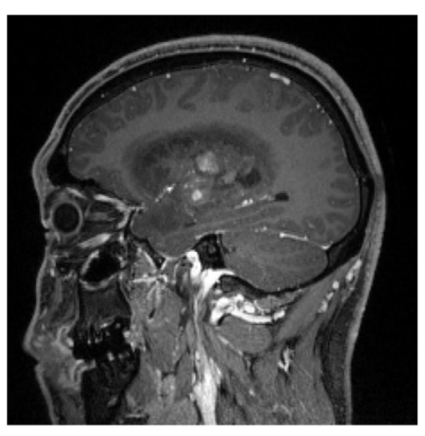
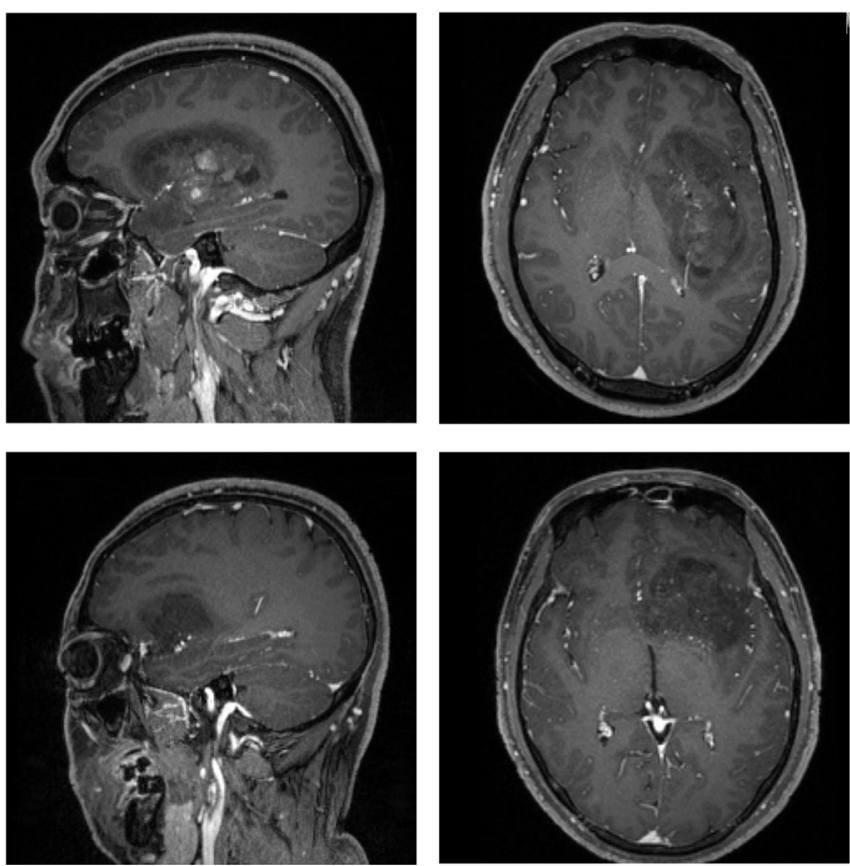

FIGURE 2 | The examples of contrast-enhanced T1 magnetic resonance images of patients with (A) LGA; (B) AA. LGA, low grade astrocytoma; AA, anaplastic astrocytoma.

\section{Classification Model Establishment}

The selection of proper features for algorithms was necessary due to the reason that excessive features could lead to inevitable overfitting. Moreover, the selected features could directly affect the diagnostic performance of the algorithms. Thus, we adopted three selection methods, including Distance Correlation, least absolute shrinkage, and selection operator (LASSO), and Gradient Boosting Decision Tree (GBDT). In the current study, the classification models were established based on three algorithms, including Linear Discriminant Analysis (LDA, also known as Fisher Linear Discriminant), Support Vector Machine (SVM), and Random Forest (RF). Then nine models were established with varied combinations of three selection methods and three classification algorithms.

For the deployment of classification algorithms, patients were divided into the training group and the validation group at a 
ratio of 4:1. For each model, sensitivity, specificity, accuracy, and area under the receiver operating characteristic curve (AUC) of the training group and the validation group were calculated to evaluate their discriminative performance. This progress was repeated 100 times to obtain the realistic distribution of accuracies. The feature selection and classification procedures were applied using Scikit-learn 0.22, a Python module for machine leaning, with parameters suggested by the developers.

\section{RESULTS}

\section{Patient Selection}

A total number of 175 patients were enrolled in the current study according to the inclusion and exclusion criteria. For patients diagnosed before 2016, we made corrections on their diagnosis based on their pathology reports according to the new WHO criteria. Ninety-five patients were diagnosed with LGA, and 80 patients were diagnosed with AA. Baseline characteristics of patients and diagnostic accuracy of human readers are shown in Table 1. For LGA patients, the median age was 32 years (range, 1-64 years), and the male ratio was 42/95 (44.2\%). And for AA patients, the median age was 39 years (range, 6-69 years), and the male ratio was $43 / 80(53.8 \%)$. The mean time between the MR scan and surgery was 6.7 and 7.2 days for LGA and AA

TABLE 1 | Baseline characteristics of patients and diagnostic accuracy of human readers.

\begin{tabular}{lccc}
\hline Characteristics & LGA (n=95) & AA (n=80) & All $(\mathbf{n = 1 7 5 )}$ \\
\hline Age (y, range) & $32(1-64)$ & $39(6-69)$ & $35(1-69)$ \\
Sex (\%) & & & \\
$\quad$ Male & $42(44.2)$ & $43(53.8)$ & $85(48.6)$ \\
$\quad$ Female & $53(55.8)$ & $37(46.2)$ & $90(51.4)$ \\
Time between MR scan and surgery (d) & 6.7 & 7.2 & 6.9 \\
Diagnostic accuracy (\%) & & & \\
Human reader 1 & 75.8 & 60.0 & 68.6 \\
Human reader 2 & 74.7 & 65.0 & 70.3 \\
\hline
\end{tabular}

LGA, low grade astrocytoma; $A$ A, anaplastic astrocytoma. patients, respectively. The mean diagnostic accuracy of the two human readers for LGA was $75.2 \%$, and that for AA was $62.5 \%$. The mean overall accuracy of human readers was $69.5 \%$.

\section{Discriminative Ability of Models}

Among the nine models we evaluated, LASSO + LDA was chosen as the optimal one with the highest AUC in the validation group. In the training group, the sensitivity, specificity, accuracy, and AUC of LASSO + LDA were 0.782, 0.717, 0.752, and 0.835, respectively; and in the validation group, the sensitivity, specificity, accuracy, and AUC were 0.751, 0.703, 0.729, and 0.825 , respectively (Table 2 ). Results showed that the diagnostic performance of LASSO + LDA was superior to human readers. The canonical discriminant functions of LASSO + LDA model for LGA, AA groups, and the group centroids are presented in Figure 3. Figure 4 is one example of the 100 validation cycles of LASSO + LDA model, which shows the distribution of the direct LDA canonical function determined for LGA and AA.

Detailed performance in the training group and validation group of all the nine models is shown in Table 2. Actually, the results suggested that all of the three algorithm-base models represented similar feasible performance when combined with a suitable selection method except for the GBDT + SVM model. The optimal SVM-based and RF-based models represented similar diagnostic performance with LDA-based models that the highest AUC of SVM-based models in the validation group was 0.808 (Table 2), and that of RF-based models was 0.821 (Table 2). However, overfitting was observed in the classification algorithm of SVM when it was combined with GBDT.

\section{DISCUSSION}

In the current study, we investigated the ability of pattern recognition techniques with radiomics features extracted from conventional MRI in discriminating atypical LGA from AA. Nine models were evaluated based on three classification algorithms and three selection methods. The results suggested that the machine learning technology could be potentially utilized in presurgical astrocytoma grading with promising ability.

TABLE 2 | Results of the discriminative models in distinguishing LGA (low grade astrocytoma) From AA (anaplastic astrocytoma) in the the training and the validation group.

\begin{tabular}{|c|c|c|c|c|c|c|c|c|}
\hline \multirow[t]{2}{*}{ Model } & \multicolumn{4}{|c|}{ Training Group } & \multicolumn{4}{|c|}{ Validation Group } \\
\hline & Sensitivity & Specificity & Accuracy & AUC & Sensitivity & Specificity & Accuracy & AUC \\
\hline Distance Correlation + LDA & 0.745 & 0.705 & 0.727 & 0.801 & 0.746 & 0.654 & 0.700 & 0.799 \\
\hline Distance Correlation + SVM & 0.659 & 0.765 & 0.707 & 0.771 & 0.721 & 0.821 & 0.766 & 0.808 \\
\hline Distance Correlation + RF & 0.761 & 0.717 & 0.741 & 0.825 & 0.770 & 0.720 & 0.740 & 0.821 \\
\hline LASSO + LDA & 0.782 & 0.717 & 0.752 & 0.835 & 0.751 & 0.703 & 0.729 & 0.825 \\
\hline LASSO + SVM & 0.713 & 0.626 & 0.673 & 0.743 & 0.756 & 0.640 & 0.706 & 0.761 \\
\hline LASSO + RF & 0.756 & 0.788 & 0.771 & 0.859 & 0.719 & 0.783 & 0.746 & 0.817 \\
\hline GBDT + LDA & 0.767 & 0.749 & 0.759 & 0.833 & 0.707 & 0.730 & 0.714 & 0.773 \\
\hline GBDT + SVM & 1 & 1 & 1 & 1 & Error & 0.537 & 0.537 & 0.5 \\
\hline GBDT + RF & 0.754 & 0.769 & 0.761 & 0.855 & 0.751 & 0.789 & 0.766 & 0.815 \\
\hline
\end{tabular}

AUC, area under curve; LDA, linear discriminant analysis; SVM, support vector machine; RF, random forest; LASSO, least absolute shrinkage and selection operator; GBDT, gradient boosting decision tree. 


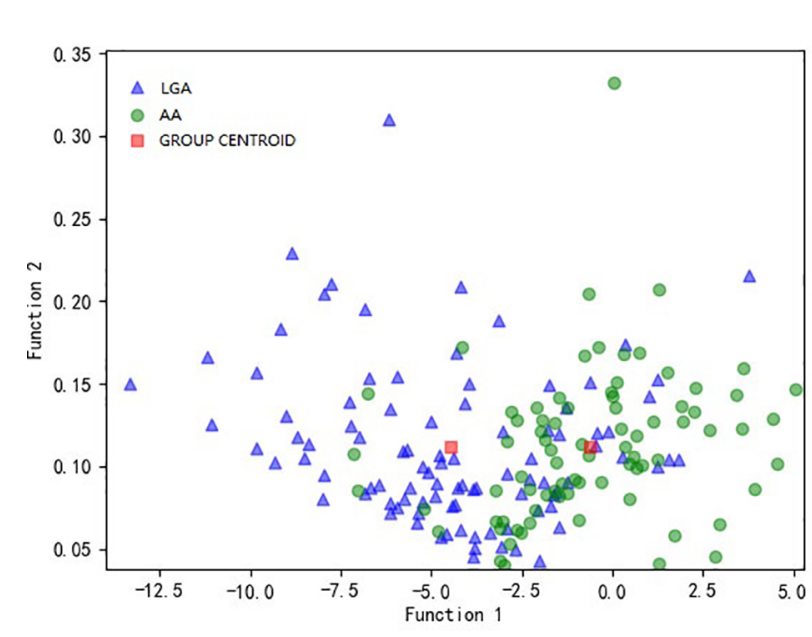

FIGURE 3 | Relationship between the canonical discriminant functions of LASSO + LDA model for LGA and AA groups and the group centroids. LASSO, least absolute shrinkage and selection operator; LDA, linear discriminant analysis; LGA, low grade astrocytoma; AA, anaplastic astrocytoma.

Astrocytoma is one of the traditional basic subtypes of diffuse grade II and III gliomas. The accurate diagnosis is clinically important because the surgical planning and therapeutic strategy are significantly different between LGA and AA. The descriptions of their characteristics on MR images are as follows: the classic LGA tumor tissues do not show enhancement after contrast administration; while AA tissues are also not usually contrast enhanced, and if they are, they represent as a focal, nodular, or patchy appearance (18). However, the enhancement pattern can also be seen on images of atypical LGA as a suggestive signal of malignant transformation and high growth rate of the tumor diameter (19). This special transforming status makes the astrocytoma grading disturbing and vulnerable when interpreting the conventional MRI, even for experienced radiologists (20).
Radiomics analysis can provide non-visual information by statistically calculating the voxels of images reflecting the tumor pathology process and abnormal microenvironment (21). It is able to transform the images into analyzable statistics with mathematical calculation, which could be further applied in machine learning technology. This set of methods has been widely explored in clinical diagnosis, tumor grading, treatment prediction, and survival prediction by previous researchers (16, 22-27). For example, the study applying RF classifier in the discrimination between primary central nervous system lymphoma and atypical glioblastoma represented satisfactory diagnostic ability with AUC of 0.921 (27). Another study applied SVM-based algorithm in glioma grading, concluding that SVM classifier feasibly achieves multiclass glioma grading as well as low classification error for intermediate (16).

However, the pervasive limitation for these studies was that the establishment of the models was seemingly arbitrary. Most studies focused on only one type of algorithms, and the adoption of feature selection method was not persuasive enough due to the lack of direct comparison. The current study established a set of machine learning-based models to discriminate LGA and AA with a relatively large number of radiomics features extracted from T1C images. The three algorithms we used in discrimination were LDA, SVM, and RF. These classification algorithms represented different types of classifiers. LDA is the classic linear classifier, which separates two or more classes by a linear combination of features (28). SVM is the classic non-linear classifier, which constructs a decision hyperplane and separates classes by maximizing the distance between the training samples of classes and the hyperplane (28). And RF is the ensemble learning classifier, which is realized by performing a weighted integration of the predictive probabilities of de-correlated trees $(29,30)$. Moreover, both LDA and SVM represent the state-ofthe-art in pattern recognition classifier applications (31). The choice of suitable algorithms is complicated. In general, as performance improves, the complexity and computational time would increase at the same time. Therefore, the researchers
A

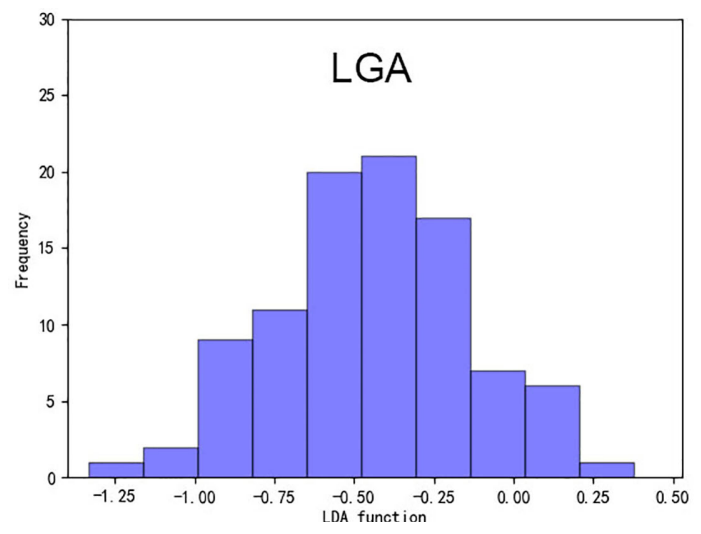

B

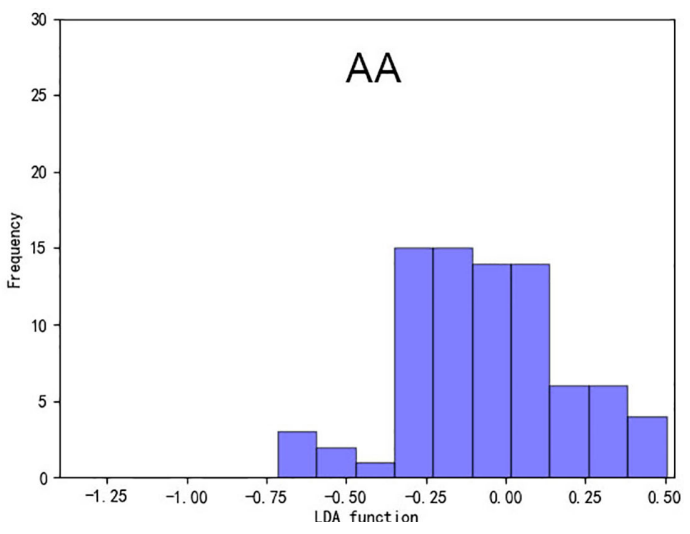

FIGURE 4 | Example of the distribution of the direct LDA canonical function determined for (A) LGA and (B) AA in one cycle. LDA, linear discriminant analysis; LGA, low grade astrocytoma; AA, anaplastic astrocytoma. 
should choose the classification algorithm based on a trade-off between performance and computational burden, especially in embedded systems (28). Based on this point, we chose three classification algorithms to make an evaluation. The results represented that the performance of all three classifiers could have similar, promising diagnostic ability when combined with suitable selection method.

As mentioned above, the selection method also played an important role in the performance of classifiers. The relatively large number of parameters made it more likely to find the optimal parameters but also increased the difficulty in feature selection. Previous studies performed feature selection with varied methods, including Student's t test with recursive feature elimination, Mann-Whitney U test with AUC of ROC, entropy-based discretization, or random forest $(27,32-34)$. We adopted three feature-selection methods (Distance Correlation, LASSO, and GBDT) in the current study.

The results of this study suggested that LASSO + LDA was the optimal discriminative model with the best performance. LASSO was recommended due to the ability of producing interpretable models when exhibiting the stability of ridge regression simultaneously. It was regarded as the nonlinear variable selection method for neural network with the advantage of minimizing the common sum of squared errors and the superior performance over other state-of-the-art variable selection methods (35). This might provide a potential underlying mechanism for our results. The LDA and RF classification algorithms both presented with consistent diagnostic performance when combining with different feature selection methods. While overfitting was observed in the model of GBDT + SVM, we suspect that the overfitting of this model was caused by the over-dependence of SVM on its kernel functions and support vectors. However, our results must be interpreted cautiously that the additional information from comparison of machine learning techniques was limited given that variance in AUC maybe partially attributed to the statistical group and all classifier/feature selection methods investigated seem perform quite comparably. Our study should be regarded as the hypothesis generation for future studies with large study population.

There were some limitations in our study. First, the study was a single-institution, retrospective study with limited patients enrolled. A research with a larger study population would be required to validate our results in the future. Second, texture features extraction was only performed on T1C images, while other conventional sequences (like T1WI, T2WI, and FLAIR) and advanced MR techniques were not investigated. Third, there was no independent validation group in our study selected from other institution. This point was not performed because that texture features could be unpredictable when extracted from images acquired with various scanners and/or protocols. Considering the analysis protocol and image processing procedure were open-source packages, the results should be validated and reproduced in a future study.

\section{CONCLUSION}

Evidence of this study indicated that radiomics-based machine learning has the potential to be utilized in the preoperative differential diagnosis between atypical LGA and AA with reliable diagnostic performance. We established high-performance prediction models based on selection methods and classification algorithms, indicating that this non-invasive approach has the potential to assist image diagnosis and aid clinical decision-making.

\section{DATA AVAILABILITY STATEMENT}

The data sets generated for this study are available on request to the corresponding authors.

\section{ETHICS STATEMENT}

Written informed consent was obtained from the individual(s), and minor(s)' legal guardian/next of kin, for the publication of any potentially identifiable images or data included in this article.

\section{AUTHOR CONTRIBUTIONS}

BC, CC, and YT designed the study. BC and CC included eligible patients, obtained medical records and MR images of each patient and performed texture analysis. JW established the models and did other statistical analysis. BC, CC, XM, and JX made substantial revisions to the manuscript. All authors contributed to the article and approved the submitted version.

\section{FUNDING}

This study was supported by 1.3.5 Project for Disciplines of Excellence, West China Hospital, Sichuan University (ZYJC18007).

\section{REFERENCES}

1. Weller M, van den Bent M, Tonn JC, Stupp R, Preusser M, Cohen-JonathanMoyal E, et al. European Association for Neuro-Oncology (EANO) Guideline on the Diagnosis and Treatment of Adult Astrocytic and Oligodendroglial Gliomas. Lancet Oncol (2017) 18(6):e315-29. doi: 10.1016/s1470-2045(17)30194-8

2. Wesseling P, Capper D. Who 2016 Classification of Gliomas. Neuropathol Appl Neurobiol (2018) 44(2):139-50. doi: 10.1111/nan.12432

3. Louis DN, Perry A, Reifenberger G, von Deimling A, Figarella-Branger D, Cavenee WK, et al. The 2016 World Health Organization Classification of Tumors of the Central Nervous System: A Summary. Acta Neuropathol (2016) 131(6):803-20. doi: 10.1007/s00401-016-1545-1

4. van den Bent MJ, Chang SM. Grade II and III Oligodendroglioma and Astrocytoma. Neurol Clin (2018) 36(3):467-84. doi: 10.1016/j.ncl.2018.04.005

5. Ostrom QT, Gittleman H, Liao P, Rouse C, Chen Y, Dowling J, et al. CBTRUS Statistical Report: Primary Brain and Central Nervous System Tumors 
Diagnosed in the United States in 2007-2011. Neuro Oncol (2014) 16 Suppl 4: iv1-63. doi: 10.1093/neuonc/nou223

6. Prados MD, Gutin PH, Phillips TL, Wara WM, Larson DA, Sneed PK, et al. Highly Anaplastic Astrocytoma: A Review of 357 Patients Treated Between 1977 and 1989. Int J Radiat Oncol Biol Phys (1992) 23(1):3-8. doi: 10.1016/0360-3016(92)90537-r

7. Piepmeier JM. Current Concepts in the Evaluation and Management of WHO Grade II Gliomas. J Neurooncol (2009) 92(3):253-9. doi: 10.1007/s11060-009-9870-z

8. Barker FG 2nd, Chang SM, Huhn SL, Davis RL, Gutin PH, McDermott MW, et al. Age and the Risk of Anaplasia in Magnetic Resonance-Nonenhancing Supratentorial Cerebral Tumors. Cancer (1997) 80(5):936-41. doi: 10.1002/ (SICI) 1097-0142(19970901)80:5<936::AID-CNCR15>3.0.CO;2-X

9. Cohen-Gadol AA, DiLuna ML, Bannykh SI, Piepmeier JM, Spencer DD. NonEnhancing De Novo Glioblastoma: Report of Two Cases. Neurosurg Rev (2004) 27(4):281-5. doi: 10.1007/s10143-004-0346-5

10. Pallud J, Capelle L, Taillandier L, Fontaine D, Mandonnet E, Guillevin R, et al. Prognostic Significance of Imaging Contrast Enhancement for WHO Grade II Gliomas. Neuro Oncol (2009) 11(2):176-82. doi: 10.1215/15228517-2008-066

11. Abrigo JM, Fountain DM, Provenzale JM, Law EK, Kwong JS, Hart MG, et al. Magnetic Resonance Perfusion for Differentiating Low-Grade From HighGrade Gliomas at First Presentation. Cochrane Database Syst Rev (2018) 1(1): Cd011551. doi: 10.1002/14651858.CD011551.pub2

12. Butowski NA, Sneed PK, Chang SM. Diagnosis and Treatment of Recurrent High-Grade Astrocytoma. J Clin Oncol (2006) 24(8):1273-80. doi: 10.1200/ jco.2005.04.7522

13. Mechtler L. Neuroimaging in Neuro-Oncology. Neurol Clin (2009) 27(1):171201, ix. doi: 10.1016/j.ncl.2008.09.015

14. De Looze C, Beausang A, Cryan J, Loftus T, Buckley PG, Farrell M, et al. Machine Learning: A Useful Radiological Adjunct in Determination of a Newly Diagnosed Glioma's Grade and IDH Status. J Neurooncol (2018) 139 (2):491-9. doi: 10.1007/s11060-018-2895-4

15. Papp L, Potsch N, Grahovac M, Schmidbauer V, Woehrer A, Preusser M, et al. Glioma Survival Prediction With Combined Analysis of In Vivo (11)C-MET PET Features, Ex Vivo Features, and Patient Features by Supervised Machine Learning. J Nucl Med (2018) 59(6):892-9. doi: 10.2967/jnumed.117.202267

16. Sengupta A, Ramaniharan AK, Gupta RK, Agarwal S, Singh A. Glioma Grading Using a Machine-Learning Framework Based on Optimized Features Obtained From T1 Perfusion MRI and Volumes of Tumor Components. J Magn Reson Imaging (2019) 50(4):1295-306. doi: 10.1002/jmri.26704

17. Nioche C, Orlhac F, Boughdad S, Reuze S, Goya-Outi J, Robert C, et al. LIFEx: A Freeware for Radiomic Feature Calculation in Multimodality Imaging to Accelerate Advances in the Characterization of Tumor Heterogeneity. Cancer Res (2018) 78(16):4786-9. doi: 10.1158/0008-5472.Can-18-0125

18. van den Bent MJ, Smits M, Kros JM, Chang SM. Diffuse Infiltrating Oligodendroglioma and Astrocytoma. J Clin Oncol (2017) 35(21):2394-401. doi: $10.1200 /$ jco.2017.72.6737

19. Rees J, Watt H, Jager HR, Benton C, Tozer D, Tofts P, et al. Volumes and Growth Rates of Untreated Adult Low-Grade Gliomas Indicate Risk of Early Malignant Transformation. Eur J Radiol (2009) 72(1):54-64. doi: 10.1016/ j.ejrad.2008.06.013

20. Law M, Yang S, Wang H, Babb JS, Johnson G, Cha S, et al. Glioma Grading: Sensitivity, Specificity, and Predictive Values of Perfusion MR Imaging and Proton MR Spectroscopic Imaging Compared With Conventional MR Imaging. AJNR Am J Neuroradiol (2003) 24(10):1989-98.

21. Hu LS, Ning S, Eschbacher JM, Gaw N, Dueck AC, Smith KA, et al. MultiParametric MRI and Texture Analysis to Visualize Spatial Histologic Heterogeneity and Tumor Extent in Glioblastoma. PloS One (2015) 10(11): e0141506. doi: 10.1371/journal.pone.0141506

22. Machine Learning Improves Diagnosis of CNS Cancers. Cancer Discov (2018) 8(5):523-4. doi: 10.1158/2159-8290.Cd-nb2018-040
23. Gaw N, Hawkins-Daarud A, Hu LS, Yoon H, Wang L, Xu Y, et al. Integration of Machine Learning and Mechanistic Models Accurately Predicts Variation in Cell Density of Glioblastoma Using Multiparametric MRI. Sci Rep (2019) 9 (1):10063. doi: 10.1038/s41598-019-46296-4

24. Liao X, Cai B, Tian B, Luo Y, Song W, Li Y. Machine-Learning Based Radiogenomics Analysis of MRI Features and Metagenes in Glioblastoma Multiforme Patients With Different Survival Time. J Cell Mol Med (2019) 23 (6):4375-85. doi: $10.1111 / \mathrm{jcmm} .14328$

25. Qian Z, Li Y, Wang Y, Li L, Li R, Wang K, et al. Differentiation of Glioblastoma From Solitary Brain Metastases Using Radiomic Machine-Learning Classifiers. Cancer Lett (2019) 451:128-35. doi: 10.1016/j.canlet.2019.02.054

26. Zhang X, Lu H, Tian Q, Feng N, Yin L, Xu X, et al. A Radiomics Nomogram Based on Multiparametric MRI Might Stratify Glioblastoma Patients According to Survival. Eur Radiol (2019) 29(10):5528-38. doi: 10.1007/ s00330-019-06069-z

27. Suh HB, Choi YS, Bae S, Ahn SS, Chang JH, Kang SG, et al. Primary Central Nervous System Lymphoma and Atypical Glioblastoma: Differentiation Using Radiomics Approach. Eur Radiol (2018) 28(9):3832-9. doi: 10.1007/s00330-018$5368-4$

28. Dellacasa Bellingegni A, Gruppioni E, Colazzo G, Davalli A, Sacchetti R, Guglielmelli E, et al. NLR, MLP, SVM, and LDA: A Comparative Analysis on EMG Data From People With Trans-Radial Amputation. J Neuroeng Rehabil (2017) 14(1):82. doi: 10.1186/s12984-017-0290-6

29. Quadrianto N, Ghahramani Z. A Very Simple Safe-Bayesian Random Forest. IEEE Trans Pattern Anal Mach Intell (2015) 37(6):1297-303. doi: 10.1109/ tpami.2014.2362751

30. Azar AT, Elshazly HI, Hassanien AE, Elkorany AM. A Random Forest Classifier for Lymph Diseases. Comput Methods Programs BioMed (2014) 113(2):465-73. doi: 10.1016/j.cmpb.2013.11.004

31. Scheme E, Englehart K. Electromyogram Pattern Recognition for Control of Powered Upper-Limb Prostheses: State of the Art and Challenges for Clinical Use. J Rehabil Res Dev (2011) 48(6):643-59. doi: 10.1682/JRRD.2010.09.0177

32. Fetit AE, Novak J, Peet AC, Arvanitits TN. Three-Dimensional Textural Features of Conventional MRI Improve Diagnostic Classification of Childhood Brain Tumours. NMR BioMed (2015) 28(9):1174-84. doi: 10.1002/nbm.3353

33. Ion-Margineanu A, Van Cauter S, Sima DM, Maes F, Sunaert S, Himmelreich U, et al. Classifying Glioblastoma Multiforme Follow-Up Progressive vs. Responsive Forms Using Multi-Parametric MRI Features. Front Neurosci (2016) 10:615. doi: 10.3389/fnins.2016.00615

34. Xiao DD, Yan PF, Wang YX, Osman MS, Zhao HY. Glioblastoma and Primary Central Nervous System Lymphoma: Preoperative Differentiation by Using MRI-based 3D Texture Analysis. Clin Neurol Neurosurg (2018) 173:84-90. doi: 10.1016/j.clineuro.2018.08.004

35. Sun K, Huang SH, Wong DS, Jang SS. Design and Application of a Variable Selection Method for Multilayer Perceptron Neural Network With Lasso. IEEE Trans Neural Netw Learn Syst (2017) 28(6):1386-96. doi: 10.1109/ tnnls.2016.2542866

Conflict of Interest: The authors declare that the research was conducted in the absence of any commercial or financial relationships that could be construed as a potential conflict of interest.

Copyright (c) 2021 Chen, Chen, Wang, Teng, Ma and Xu. This is an open-access article distributed under the terms of the Creative Commons Attribution License (CC BY). The use, distribution or reproduction in other forums is permitted, provided the original author(s) and the copyright owner(s) are credited and that the original publication in this journal is cited, in accordance with accepted academic practice. No use, distribution or reproduction is permitted which does not comply with these terms. 